\title{
Route Optimization in logistics distribution based on Particle Swarm Optimization
}

\author{
Appiah Martinson Yeboah \\ School of Management Jiangsu University
}

\author{
Xiong Qiang \\ School of Management Jiangsu University
}

\begin{abstract}
Vehicle Routing Problem (VRP) addresses a problem which identifies routes scheduled for vehicles moving from a distribution center to serve specific customers and returns to the distribution center. Notwithstanding, cost associated with transportation of business have drawn much attention in the past few years owing to the recent rise in fuel prices, therefore this paper study's the problem of routing in cold chain logistics distribution with the goal of minimizing the total transportation cost. In this paper a single objective model is formulated and then solved by the Particle Swarm

Optimization algorithm. A computational experiment is carried by the proposed model to obtain optimal distance and imputed in to the cost function to obtain the optimal cost. We found that an increase in population size and the number of iterations gives better minimization results.
\end{abstract}

\section{Keywords}

Particle Swarm Algorithm, Vehicle Routing Problem, Capacitated Vehicle Routing Problem

\section{INTRODUCTION}

The Vehicle Routing Problem (VRP) usually occurs when there is movement of goods from a depot to serve specific customers dispersed and taking into consideration how cost associated with the distribution can be minimized at an increased net income. It is widely applied to various areas such as transport delivery routing, urban school bus route planning, mail delivery, rural school bus routing planning, trucks that deliver gasoline urban trash collection and snow plough and (Shahrzad Amini, 2010). This VRP is a variant of the well-known routing problem called Capacitated Vehicle Routing Problem (CVRP). This problem helps to identify routes which starts and ends at a distribution center. Mathematically it can be represented as a set vehicles of the same kind (V) which has a capacity (Q). It must satisfy the demand set of customers (N). It has been designed such that a vehicle visits a customer once. The total demand of the customer on a particular route must not exceed the vehicle capacity $\mathrm{Q}$. The goal is to minimize the distribution cost for the performed routes. In this paper the PSO model is designed to obtain optimal distance and a transport objective is

modeled to minimize transport cost in cold chain logistics considering a Capacitated Vehicle Routing Problem.

VRP is an interesting subject which belongs to a category of combinatorial optimization problems. Dantzig (1959) first proposed the truck dispatching problems. Theoretically, this is an NP-hard problem, which means that it is believed that one may never find a computational technique that guarantees an optimal solution to larger instances for such problems. Researchers have developed many solution approaches to solve VRPs, such as exact algorithms and heuristic algorithms (Cordeau, 2007). Meta-heuristics are a kind of heuristics, which have widely been applied to VRPs. The performance of meta-heuristics is often better than classical heuristics. Popular metaheuristics include Genetic Algorithm (GA), Tabu Search (TS), Simulated Annealing (SA), Ant colony systems (ACS), Particle Swarm Optimization (PSO), Scatter Search (SS) etc. (Chen,2011).

However, this paper will consider the use of the Particle Swarm Optimization to solve the VRP problem. PSO is a population-based search method proposed by Kennedy and Eberhart (Kennedy, 1995) , which is motivated by the group organism behavior such as bee swarm, fish school, and birds flock. PSO imitate the physical movement of the individuals in the swarm as a searching method. Therefore, to resolve this NP-hard problem, the section below reviews related articles which have used PSO to solve the VRP variants.

Xiao (2005) proposed a modified particle swarm optimization for solving the VRP. He also applied a method based on PSO to solve the discrete VRP. They changed the VRP into a quasi-continuous problem by designing a new real coding and solved it using PSO. Zhang (2006) used a combination of PSO and Simulated Annealing (SA) for solving the VRP which can avoid being trapped in a local optimum using probability search and the computation results revealed that it was better.

A new hybrid approximation algorithm to solve CVRP was introduced by Chen (2006), in which discrete particle swarm optimization combines global search and local search to search the optimal results and SA uses certain probability to avoid being trapped in a local optimum. Zhang (2010) designed a new particle swarm optimization algorithm, which introduces the local neighbor mechanism and can optimize infeasible solutions. The algorithm obtains comparatively satisfactory results in solving the vehicle routing problem with time window. Dongqing Ma (2014) established a two-way vehicle scheduling problem model with the basis of the particle swarm algorithm and the mountain climbing operation is introduced, which effectively solves the problem of logistics distribution.

$\mathrm{Xu}$ (2007) solved the VRP with multiple objectives by combining PSO with mutation operator and with the help of roulette-wheel. In the solution, the discrete problem converged to optimal Pareto set and equally distributed along the pareto curve after the Pareto muster was evaluated with relative minimum distance method. Experiment showed that the algorithm was simple and effective. Ahmed (2016) introduced a mixed-integer concave minimization problem in supply chain of cold chain to reduce total cost including capacity, transportation, inventory costs and the global warming impact. The method was checked through extensive 
numerical testing on two realistic case studies from different industries, and the results show that it is possible to substantially minimize global warming effect of cold supply chains with a small increase and sometimes with virtually no increase in cost

Ali (2014) considered transportation unit capacity and storage unit capacity and addressed a new inventory model that considers both cost and emission functions in cold chain environment. Kumar (2016) considers production and pollution routing problems with time window (PPRPTW), used a hybrid self-learning Particle Swarm Optimization (SLPSO) algorithm in the multi-objective framework to solve the problem. A Bilayer local Researchbased Particle Swarm Optimization (BLS-PSO) with a novel decoding approach was developed by (Sun, 2018) to solve a CVRP. In the Bilayer local search, a layer of the local search is representing a whole population in iteration while the other is used on a collection of the best particles generated in different generations. Results showed that the BLS-PSO performs better than other PSO-based methods

Marinakis (2015) also presented a new version of the particle swarm optimization (PSO) algorithm suitable for discrete optimization problems and applied it for the solution of capacitated location routing problem and for the solution of a new formulation of the location routing problem with stochastic demands.

In short based on the reviewed literatures, there are many researches on VRP however to the best of our knowledge few have to use the PSO model to optimize distance routes and minimize cost in cold chain logistics considering a Capacitated Vehicle Routing Problem. Hence this paper proposes the Particle Swarm Optimization model to determine shortest distance and finally optimize transport cost. Finally, the validity and feasibility of the algorithm is verified by computational results and its benefits.

The paper is organized as follows. The literature associated to the Capacitated Vehicle Routing Problem and the methods used by other authors to solve the VRP is described in Section II. Section III displays the method and model used to solve the VRP. It also presents a detailed description of the framework used by the proposed algorithm. A computational result on the optimal distance and minimized transport cost is reported in Section IV. Section V. Finally contains concluding remarks and future research.

\section{MODEL FORMULATION}

\subsection{Problem Assumptions}

a. Customers demand and capacity of vehicles are known.

b. There is only one distribution center where goods are loaded for distribution.

c. Transportation cost of distribution vehicle comprises of two parts that is the fixed cost and variable cost.

d. The totality of all vehicles which are the same type is fixed in the distribution.

e. Assuming Depot A coordinates and the customers are serviced by a number of vehicles.

\subsection{Mathematical Model}

\subsubsection{Transport Cost}

The transportation costs of vehicles are influenced by fuel consumption, maintenance and other factors, and it is proportional to the vehicle mileage. The transportation costs in the model can be expressed as:

$$
\sum_{i=0}^{L} \sum_{j=0}^{L} C_{i j} * X_{i j}
$$

St.

$$
X_{i j}=0 \text { or } 1, i j=1,2,3 \ldots l
$$

\section{PARTICLE SWARM OPTIMIZATION (PSO) DESIGN}

The Particle Swarm optimization is inspired by a simulation of social behavior of birds within a flock. This is an evolutionary computation technique invented by Eberhart and Kennedy. It is a population-based stochastic approach for solving continues and discrete optimization problems. In this method simple software agents called particles move in the search space of an optimization problem (Anita Tandan, 2013). In the PSO, individuals are particles and are flown through hyper dimensional search space. They Simulate birds swarm behavior and made each particle in the swarm move according to its experience and the best experience of particle. Each particle represents a potential solution to problem and searches around in multidimensional search space. (Kang Li1, 2016). It widely applied fields like function optimization, neutral network and other application

The pseudo code of the PS is as follows:

For each particle

Initialize particle

End

Do

For each particle

Calculate fitness value

If fitness value is better than the best fitness

value(pbest) in history

Set current value as the new pbest

End

Choose the particle with the best fitness value of all the particles as the gBest

For each particle

Calculate particle velocity according to equation

Update particle position according to equation

End

Step 1: PSO Algorithm parameter Settings

Set 2: Initialize the population. Initialize the position and particle velocity of particles and calculate particle fitness value according to fitness function, $\mathrm{N}=100$

Step3: look for initial extremum and group extremum according to the initial particle fitness value 


\section{Step4: iterative optimization}

Step 5: Update velocity vector of each particle

Each particle represents a candidate solution and the quality of solution is determined by the optimization objective function. The computation formula of iterative optimization is a follow:

$$
\begin{gathered}
V_{i}^{t+1}=W^{t} V_{i}^{t}+C_{1} r_{1}\left(p B e s t_{i}^{t}-X_{i}^{t}\right)+C_{2} r_{2}\left(g B e s t^{t}\right. \\
\left.-X_{i}^{t}\right)
\end{gathered}
$$

Step 6: Update each particle position vector

$$
X_{i}^{t+1}=X_{i}^{t}+V_{t}+1_{i}
$$

$\mathrm{W}$ is inertia weight, $\mathrm{i}=1,2, \ldots, \mathrm{n} ; \mathrm{t}$ represents the current iterations times; $V_{i}$ represents speed; $C_{1}$ and $C_{2}$ are two positive constants, $r_{1}$ and $r_{2}$ are uniformly distributed random number in $[0,1]$.

Step 7 Interpretation of results

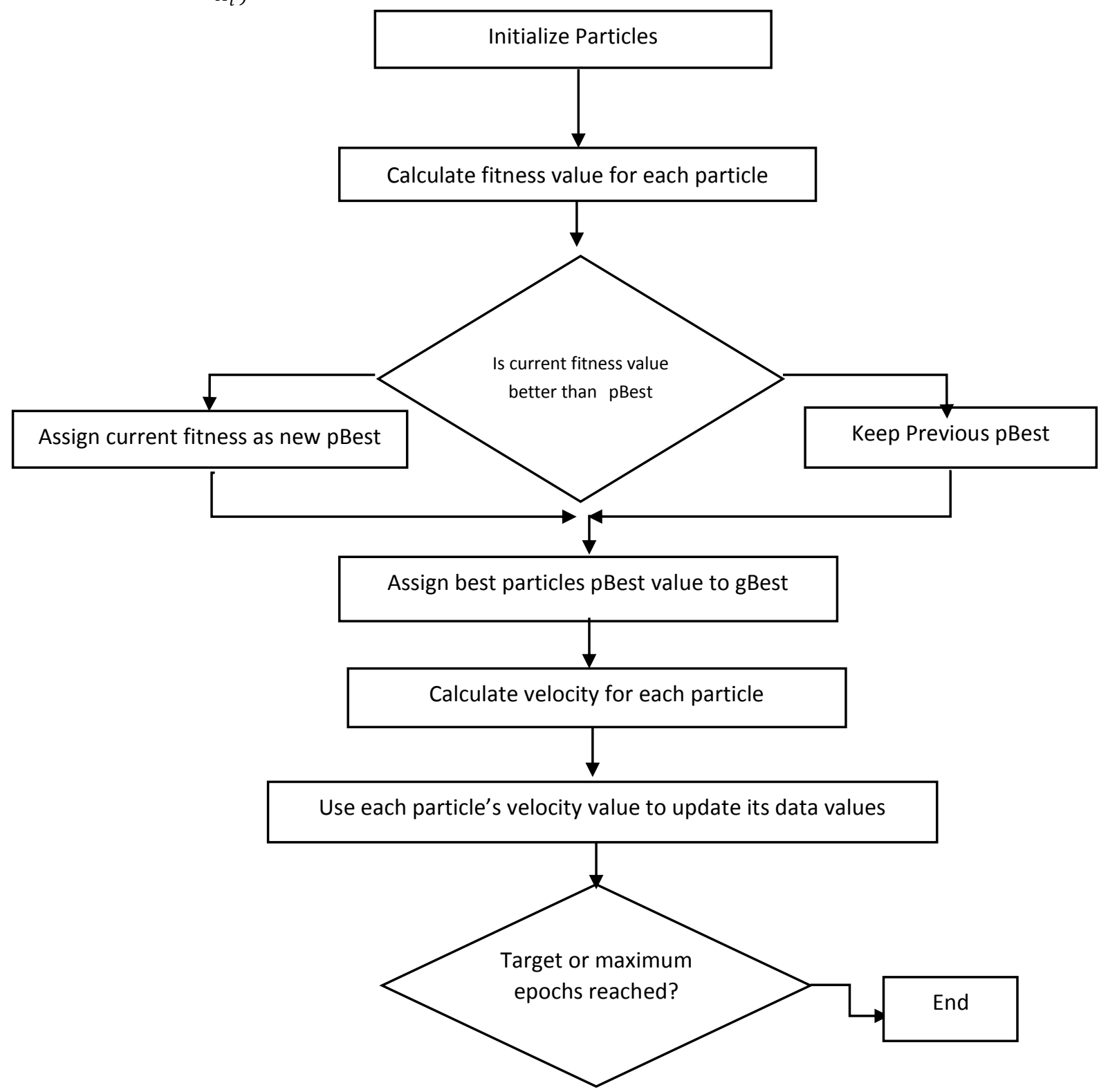

Fig 1. Flow chat of Particle Swarm Optimization

\section{EXPERIMENTAL DESIGN AND COMPUTATIONAL RESULTS}

The researchers used data set from (Songyi Wang, 2017) and (Shahrzad Amini, 2010) which considers a fixed distribution center distributing goods to 100 customers, a fixed vehicle cost of 200 Yuan and fuel cost of 3 Yuan $/ \mathrm{km}$ per mileage in the transportation process and for the Parameters. The key parameters used in this study for the PSO are presented in Table1.This paper uses MATLAB R2014a to implement the PSO and all experiments in this are evaluated on PCs with intel ${ }^{\circledR}$ Core $^{\mathrm{TM}}$ (Santa Clara, CA,USA) i7-3610QM CPU@2.10GHz $2.10 \mathrm{GHz}$ and memory of $4 \mathrm{~GB}$. 
Table 1. PSO Parameters setting

\begin{tabular}{cc}
\hline Parameter & Value \\
\hline Population (NP) & 20 to 100 particles \\
Number of iterations & 100 \\
Inertia weight & $W_{\text {max }} 0.9, W_{\min }=0.1$ \\
Acceleration factors & $C_{1}=2, C_{2}=2$ \\
Initial Velocity & 0.1 \\
\hline
\end{tabular}

Table 2. Data Set

\begin{tabular}{cc}
\hline Item & Value \\
\hline Transport cost in per unit mileage & 3 Yuan $/ \mathrm{km}$
\end{tabular}

fixed cost of per vehicle

200 Yuan

Source: Data set from (Songyi Wang, 2017)

\subsection{Computational results from Simulation and Analysis}

In this work MATLAB was used to implement the PSO algorithm. After 101 times of iti conducted, the solution was stable. PSO was used because it helps to obtain the value which is close to the actual optimal function value. This shows that the PSO has a stronger function extreme value optimization ability. Finally, in figure 2, the researchers obtained an optimal individual fitness value as 317.1429 at average time of $7.328950 \mathrm{~s}$

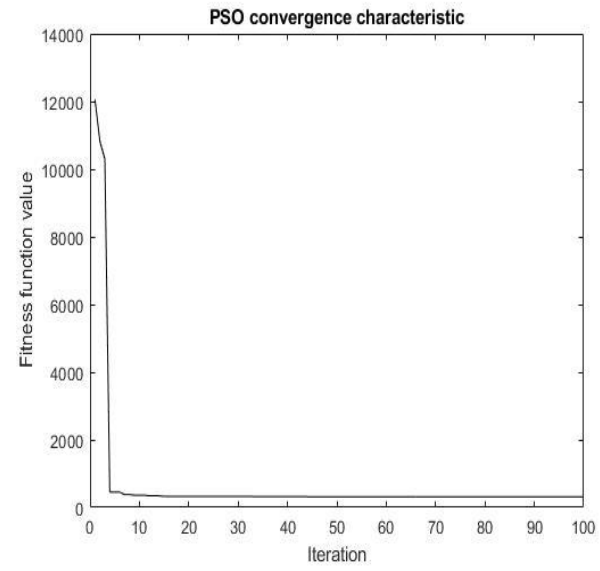

Fig 2. Best individual fitness value

From the figure, it is shown that an optimal individual fitness value of 317.1429 was obtained.

In the beginning, the fitness of PSO declined to the best value much faster and later converges at point 317.1429.

Operational results are shown below

Optimal distance $=317.1429$

Optimal cost $=317.1429 * 3+200=1151.4287$

\section{CONCLUSION AND FUTURE WORKS}

In conclusion, this paper considers the particle swarm optimization algorithm (PSO) model to solve Capacitated
Vehicle Routing Problem by identifying the shortest distance and optimal cost. In the experiments, parameters such as the population size of the algorithm and number of iterations had effect on the optimization result. In the experiment, it was showed that when the population size increased, optimal and operational results can be obtained faster and better. Also, by increasing the number of iterations, the result was getting better and searching the excellent solution to the increasingly high probability. When the population size reached to a certain level, the results of the optimization stabilized at (317.1429) which is the individual fitness value and did not changed significantly for longer time even with the increase of the population size. Also, an optimal transport cost of 1151.4287Yuan was obtained. In this paper it could be seen that the structure and mechanism of the proposed PSO may be further optimized and since these efforts may yet contribute to additional performance gains in both the solution quality and computational time, a further study on these aspects is still necessary. Therefore, for future research, time window for vehicle routing can be looked at to form routing considering loading and unloading time, real-time constraints and also the reduction of the emission rates of $\mathrm{Co} 2$ can be carried out to show generality of the method.

\section{ACKNOWLEDGEMENTS}

This work is supported by the MOE Layout Foundation of Humanities and Social Sciences [NO.16YJA630001].

\section{REFERENCES}

[1] Ali B., J. P. (2014). A new inventory model for cold items that considers costs and emissions. Int. J. Production Economics, 155

[2] Ali B., J. P. (2014). A new inventory model for cold items that considers cost and emissions. International Journal Production Economics , 114-125.

[3] Anita Tandan, R. R. (2013). A Survey on Particle Swarm Optimization Methods for Image Segmentation. Computer Science and Engineering , $1-6$.

[4] Chen, A. Y. (2006). Hybrid discrete particle swarm optimization algorithm for capacitated vehicle routing problem. Journal of Zhejiang University Science A, 607-614.

[5] Chen, Y. K. (2011). A HYBRID PSO ALGORITHM FOR THE CVRP PROBLEM. In Proceedings oftheInternationalConferenceonEvolutionaryComputa tionTheoryandApplications (pp. 539-543). Taipei, Taiwan: SCITEPRESS

[6] Cordeau, J. F. (2007). Handbook in Operations Research and Management Science(.

[7] Dongqing Ma, W. W. (2014). Logistics distribution vehicle scheduling based on improved particle swarm optimization. Computer Engineering and Applications,, 50(11): 246-270.

[8] E., A. S. ( 2016). . Cold supply chain design with environmental considerations: A simulationoptimization approach. . European Journal of Operational Research,,, 251.

[9] Kang Li1, J. Z. (2016). A new discrete particle swarm optimization for location inventory routing problem 
in cold logistics. Revista de la Facultad de Ingeniería U.C.V., 89-99.

[10] Kennedy, J. a. (1995). Particle Swarm Optimization. proceedings of IEEE international conference on neural networks, (pp. 1942-1948).

[11] Kumar, K. D. (2016). Multi-objective modeling of production and pollution routing problem with time window: A self-learning particle swarm optimizaion approach . Computers and Industrial Engineering 29-40.

[12] Shahrzad Amini, H. J.-M. (2010). A PSO APPROACH FOR SOLVING VRPTW WITH REAL CASE STUDY. IJRRAS, 1-10.

[13] Songyi Wang, F. T. (2017). Optimization of Vehicle Routing Problem with Time windows for Cold Chain Logistics Based on Carbon Tax. sustainability, 1-24.

[14] Sun, A. K. (2018). Bilayer Local Search Enhanced Particle Swarm Optimization for the Capacitated Vehicle Routing Problem. . Algorithms, , 11(3), 1-22.
[15] Xiao, J. M. (2005). particle swarm optimization algorithm for vehicle routing problem. Computer Integrated Manufacturing Systems, 577-581.

[16] Xu, J. a. (2007). Hybrid particle swarm optimization for vehicle routing problem with multiple objectives. Computer Integrated Manufacturing SystemsBeijing,, Vol. 13, No. 3, pp. 573.

[17] Y, M. (2015). An Improved Particle Swarm Optimization Algorithm for the capacitated location routing problem and for the location routing problem with stochastic demands. Applied Soft Computing, 680-701.

[18] Zhang, L. P. (2006). A hybrid particle swarm optimization algorithm for vehicle routing problem with time windows2. Journal of Shanghai Jiaotong University, 1890.

[19] Zhang., Y. W. (2010). Modified Particle Swarm Optimization algorithm for vehicle routing problem with time. Computer Engineering and Applications, 46(15): 230-234. 